\title{
Intraoperative computed tomography of a resected lung inflated with air to verify safety surgical margin
}

\author{
Go Kamimura ${ }^{1,2}$, Kazuhiro Ueda ${ }^{2}$, Soichi Suzuki ${ }^{2}$, Koki Maeda $^{2}$, Hiroto Hakamada ${ }^{3}$ Masami Sato $^{2}$ \\ ${ }^{1}$ Department of General Thoracic Surgery, Nanphu Hospital, Kagosima, Japan; ${ }^{2}$ Department of Radiology, Nanphu Hospital, Kagosima, Japan; \\ ${ }^{3}$ Department of General Thoracic Surgery, Kagoshima University Graduate School of Medical and Dental Sciences, Kagoshima, Japan
}

Contributions: (I) Conception and design: G Kamimura, M Sato; (II) Administrative support: None; (III) Provision of study materials or patients: None; (IV) Collection and assembly of data: G Kamimura, K Ueda; (V) Data analysis and interpretation: G Kamimura, K Ueda; (VI) Manuscript writing: All authors; (VII) Final approval of manuscript: All authors.

Correspondence to: Kazuhiro Ueda, MD, PhD. Department of General Thoracic Surgery, Kagoshima University Graduate School of Medical and Dental Sciences, 8-35-1 Sakuragaoka, Kagoshima 890-8520, Japan. Email: k7433286@kadai.jp.

Background: During sublobar resection for small, indistinct lung cancer, surgeons may be uncertain as to whether or not the target lesion has been resected and the surgical margin is sufficient. We herein report our procedure for confirming the success of sublobar resection without incising the resected specimen.

Methods: We reviewed our initial experience of 12 patients with intrapulmonary lesions (consolidation diameter $\leq 1 \mathrm{~cm}$ ) who underwent thoracoscopic pulmonary wedge resection using autostapler. Six patients had primary adenocarcinoma showing part solid lesion, and remaining six patients had metastatic carcinoma showing purely solid lesion. Intraoperatively, the resected specimen was inflated with air and subjected to computed tomography (CT). The maximum tumor diameter and surgical margin length were measured intraoperatively on CT and postoperatively on formalin-fixed specimen. Surgical stump cytology was also done to verify surgical margin.

Results: According to the intraoperative CT, complete resection was confirmed in all patients. The intraoperative CT-based maximum tumor diameter closely correlated with the macroscopically measured one $(\mathrm{r}=0.971, \mathrm{P}<0.0001)$. However, the tumor shrunk after formalin-fixation by $16.0 \%$ in patients with primary lung cancer $(\mathrm{P}<0.01)$, but not in patients with metastatic lung cancer. The intraoperative CT-based margin length closely correlated with the macroscopically measured one $(r=0.984, P<0.0001)$. However, the margin shrunk after formalin-fixation in both patients with primary lung cancer and metastatic lung cancer, by $15.1 \%$ and $15.7 \%$, respectively. Stump cytology was negative in all patients. Consequently, no recurrence was found during postoperative follow-up of 23 months (range, 14-31 months).

Conclusions: Intraoperative CT is reliable for diagnosing the presence of a target lesion within the resected specimen as well as for estimating the surgical margin length in patients undergoing sublobar resection for intrapulmonary indistinct lesions.

Keywords: Lung cancer; intraoperative CT; surgical margin

Submitted May 26, 2021. Accepted for publication Sep 22, 2021.

doi: 10.21037/qims-21-562

View this article at: https://dx.doi.org/10.21037/qims-21-562 


\section{Introduction}

The incidence of sublobar resection for small, indistinct pulmonary nodules is increasing because of the increased frequency of performing computed tomography (CT)based whole lung screening. Various techniques for the intraoperative localization of such indistinct lesion have been reported $(1,2)$. However, surgeons still worry about whether or not the lesion is actually included in the resected specimen and if the surgical margin is sufficient, particularly if the lesion is impalpable or accompanied by ground glass component. Although cutting the specimens into pieces can help identify the lesions, it prevents the subsequent pathological assessment of the invasiveness of primary lung adenocarcinoma (invasive adenocarcinoma, minimally invasive adenocarcinoma, or adenocarcinoma in situ) and the accurate measurement of the surgical margin. Sawabata et al. reported that the shortage of the surgical margin in pulmonary wedge resection for lung cancer likely results in positive surgical stump cytology, leading to local recurrence $(3,4)$. They advocated that the surgical margin of greater than the maximum tumor diameter was optimal to prevent local recurrence (3). Mohiuddin et al. also reported the importance of surgical margin length in pulmonary wedge resection for lung cancer: they did not find any postoperative local recurrence if surgical margin was more than $15 \mathrm{~mm}$ (5). Therefore, $15 \mathrm{~mm}$ (if the tumor is greater than $15 \mathrm{~mm}$ ) or greater than the maximum tumor diameter is regarded as sufficient surgical margin in patients undergoing sublobar resection. However, the previous studies mainly subjected tumors greater than $1 \mathrm{~cm}$. Furthermore, the necessary surgical margin in adenocarcinoma in situ or minimally invasive adenocarcinoma, showing ground glass component, remains unclear probably because, in practice, it may be difficult to accurately measure the surgical margin in subcentimeter tumor or tumors with ground glass component.

Currently, we scanned the resected specimens, which was inflated with air, immediately after pulmonary wedge resection in patients with consolidation diameter of $1 \mathrm{~cm}$ or less. We hypothesized that this technique is effective in identifying if the tumor is indeed resected or not, as well as in estimating the real surgical margin length. We believe that our method may be useful in studying the optimal surgical margin required in tumors with ground glass component.

We present the following article in accordance with the MDAR checklist (available at https://dx.doi.org/10.21037/ qims-21-562).

\section{Methods}

\section{Patients}

The study was conducted in accordance with the Declaration of Helsinki (as revised in 2013). This study was approved by the institutional review board of Kagoshima University Graduate School of Medical and Dental Sciences (Approval No. 210025), and individual consent for this retrospective analysis was waived. We performed our novel technique in 12 patients who underwent thoracoscopic pulmonary wedge resection for metastatic lung cancer $(n=6)$ or primary lung cancer $(n=6)$, with a consolidation size $\leq 1 \mathrm{~cm}$, between October 2018 and March 2020 in Nanpu Hospital. All patients underwent wedge resection using endoscopic stapler (Covidien, Minneapolis, MN, USA) for curative intent: we basically perform pulmonary wedge resection in patients with metastatic lung cancer, less than $3 \mathrm{~cm}$ in maximum diameter, or primary lung cancer, showing ground glass attenuation and less than $1 \mathrm{~cm}$ in consolidation diameter. All patients had solitary pulmonary nodule without any abnormal uptake in other areas on ${ }^{18}$ F-fluorodeoxyglucose positron emission tomography. Preoperative bronchoscopic biopsy was omitted in the 12 patients due to the tumor location or small tumor size. Primary organ in six patients with metastatic lung cancer was colon $(n=4)$, small intestine $(n=1)$, or bile duct $(n=1)$. Intraoperatively, the tumor location was identified either by finger palpation $(n=9)$ or by intraoperative transthoracic marking with pyoktanin blue solution $(n=3)(6)$.

\section{The radiological assessment}

The resected specimens were inflated by the careful instillation of air. Puncture was performed at a distant site, where the pulmonary lesion was absent, using a 23-G puncture needle connected to an appropriately sized syringe. The instillation was done slowly in order to prevent alveolar wall destruction. The degree of inflation differs among specimens because air leak from the puncture site or stapled site eventually develops. After the specimen was placed in a plastic container, the specimen along with the container underwent conventional 64-detector CT (Revolution GSI; GE, Chicago, IL, USA) as soon as possible. The scan parameters were as follows: tube voltage, $80 \mathrm{kV}$; rotation time, $0.6 \mathrm{~s}$; beam width, $20 \mathrm{~mm}$; pitch, 1.375 ; slice thickness, $0.625 \mathrm{~mm}$; slice interval, $0.625 \mathrm{~mm}$; scan field of view (FOV), $8 \mathrm{~cm}$; matrix, $512 \times 512$; recon type, $\mathrm{HD}$ Lung; noise index, 10. It generally takes 5 minutes or less 

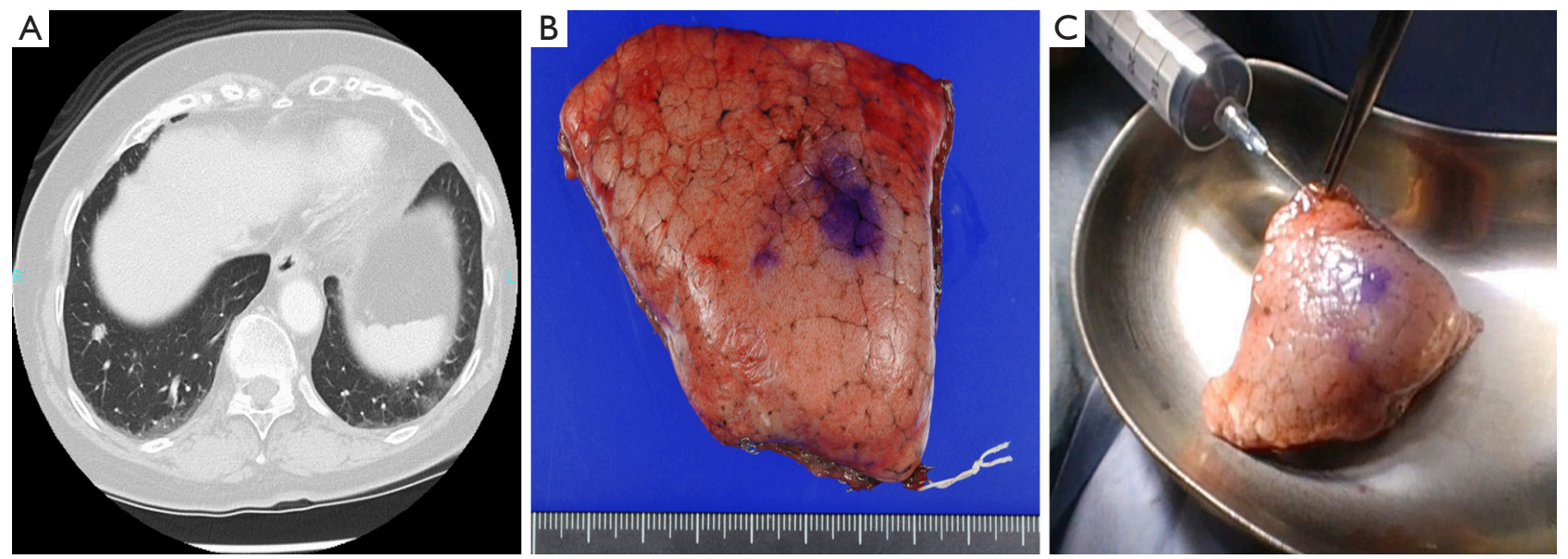

Figure 1 Representative case of a 77-year-old woman with primary lung adenocarcinoma. Chest computed tomography shows subpleural part solid lesion in the right lower lobe (A). The patient underwent wedge resection of the right lower lobe with an endoscopic stapler (B). The resected specimen was inflated by the slow instillation of air so as not to destroy the normal alveolar wall structure (C). Note that the puncture was done at a site distant from the pulmonary lesion using a 23-G puncture needle connected to an appropriately sized syringe.

for scanning of the resected specimen and image acquisition. The obtained images were evaluated intraoperatively in the operating room on multi-planner reconstruction images. Figures 1-3 show two representative cases with primary lung cancer.

\section{The pathological assessment}

We routinely performed irrigation of any cartridges used in the pulmonary stapling in order to make a rapid cytological diagnosis for surgical stump positivity (7). After intraoperative CT, the specimen was instilled with $10 \%$ formalin neutral buffer solution using a 23-G needle and then soaked in the solution for $48 \mathrm{~h}$. After removing the stapler needles from the staple line, the specimen was cut into pieces for macroscopic and microscopic evaluations of the tumor diameter and surgical margin. Maximum tumor diameter and surgical margin length were macroscopically measured on the formalin-fixed specimen by an attending pathologist after making a section with reference to the intraoperative CT of the specimen. In order to evaluate the validity of intraoperative $C T$, we prospectively recorded the maximal tumor diameter on preoperative and intraoperative CT and by direct vision of the resected specimen, and we also recorded the surgical margin length on intraoperative CT and direct vision on the resected specimen. CTbased measurements were done by two authors (KG and $\mathrm{SM}$ ), independently. The final decisions were reached by consensus.

\section{Statistical analysis}

Pearson's correlation analysis was performed to analyze the correlation between the parameters measured on preoperative $\mathrm{CT}$ and those measured on intraoperative $\mathrm{CT}$, as well as between the parameters measures on intraoperative CT and those measured on direct vision. Paired $t$-test was used to compare tumor diameters determined by CT and those determined by direct vision, as well as to compare surgical margin determined by CT and those determined by direct vision. $\mathrm{P}$ values of $<0.05$ were considered to indicate statistical significance. The statistical analyses were performed using the SPSS software program (SPSS statistics version 22; IBM, USA).

\section{Results}

The clinical characters of the 12 patients are shown in Table 1 . After wedge resection, the target lesions were identified within the specimens in all 12 patients on intraoperative CT, resulting in the measurement of the maximal tumor diameter and the surgical margin. We intended to secure a surgical margin of at least the same distance as the consolidation diameter of the tumor on preoperative CT. As a result, the surgical margin was successfully secured in all patients except for one (Case 10, Table 1). In this case, additional resection 

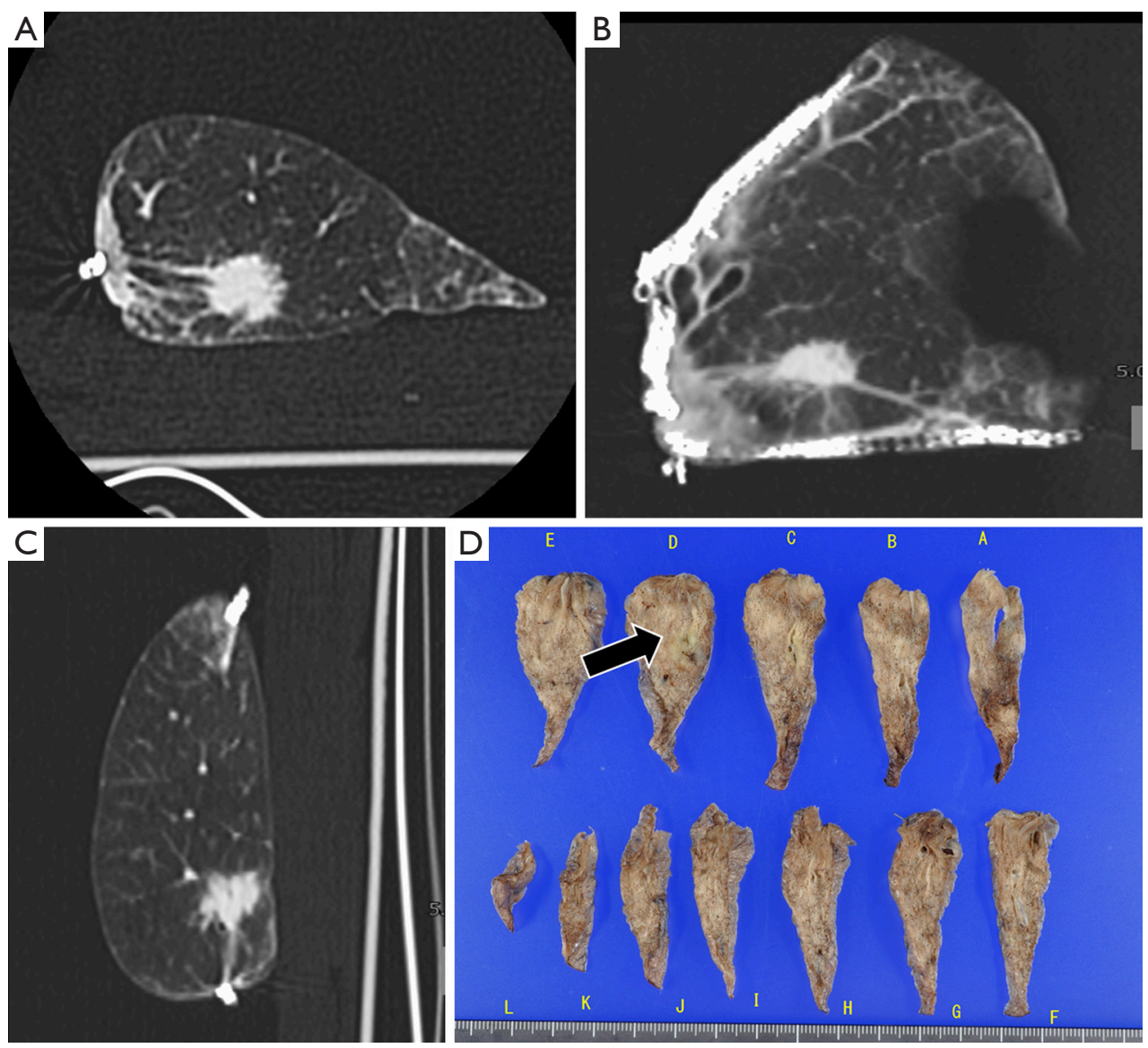

Figure 2 Radiological and macroscopic findings of the resected lung in the patient with primary lung adenocarcinoma in the right lower lobe (same patient as Figure 1). After the excised specimen was inflated, the specimen immediately underwent conventional 64-detector computed tomography (A-C). The multiplanar reconstruction images of the resected specimen facilitated the accurate measurement of the maximal tumor diameter and minimal surgical margin. Forty-eight hours after the fixation of the specimen with $10 \%$ formalin solution, the stapler needles were removed from the stapled site, and the specimen was cut into pieces for macroscopic and microscopic evaluations (D). The tumor revealed to be invasive adenocarcinoma with invasive size of $6 \mathrm{~mm}$.

was abandoned because of the anatomical difficulty and pulmonary functional limitation. The irrigation cytology, as a substitute for stump cytology, was negative in all the patients. Consequently, no recurrence was found in the hemithorax during the median postoperative follow-up of 23 months (range, 14-31 months).

According to a correlation analysis, the maximal tumor diameter on intraoperative CT was found to be closely correlated with that on preoperative CT in overall patients (Figure 4A). According to a paired $t$-test, the maximal tumor diameter on preoperative CT was not significantly different from that on intraoperative CT in both patients with primary lung cancer and metastatic lung cancer (Figure $4 B$ ).
Likewise, according to a correlation analysis, the maximal tumor diameter on intraoperative CT was found to be closely correlated with that on direct vision in overall patients (Figure $5 A$ ). However, according to a paired $t$-test, there was a significant discrepancy between the maximal tumor diameter on intraoperative CT and that on direct vision in patients with lung cancer (Figure 5B).

With regard to the surgical margin, although the length on intraoperative CT correlated closely with that on direct vision of the resected specimens in overall patients (Figure $6 A$ ), the margin on direct vision was significantly shorter than that on intraoperative CT in both patients with primary lung cancer and metastatic lung cancer (Figure 6B). 

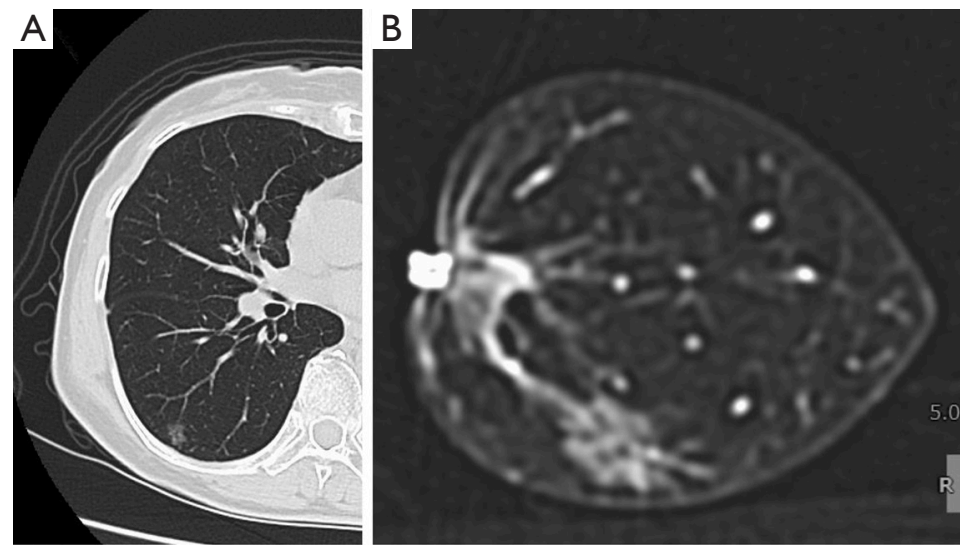

Figure 3 Representative case of a 61-year-old woman with primary lung adenocarcinoma. Chest computed tomography shows subpleural purely ground glass lesion in the right lower lobe (A). The patient underwent wedge resection of the right lower lobe with an endoscopic stapler under transthoracic marking because the tumor was not palpable. Although the tumor showed purely ground glass attenuation, the tumor is clearly depicted by intraoperative computed tomography (B). The tumor revealed to be adenocarcinoma in situ.

Table 1 Patient characteristics $(\mathrm{n}=12)$

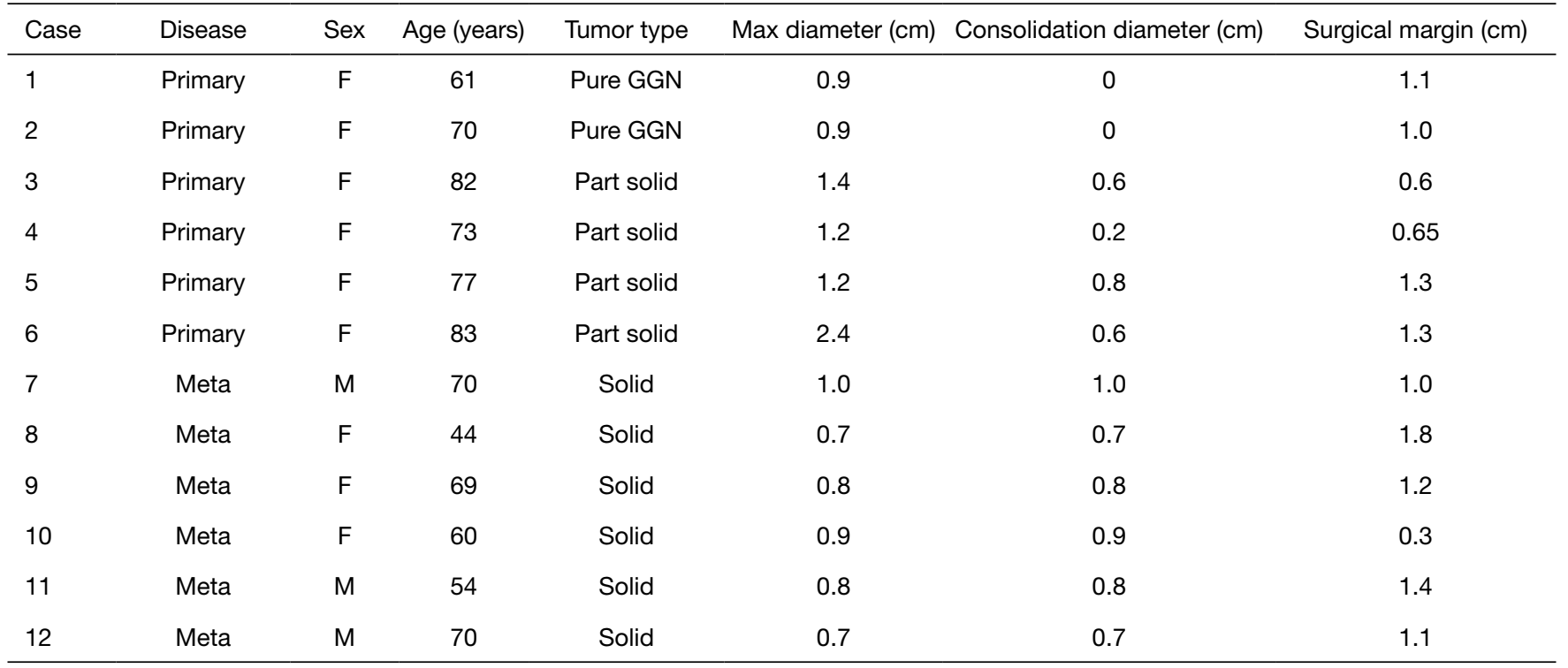

The surgical margin was measured on direct vision of the resected specimen. Primary, primary lung cancer; Meta, metastatic lung cancer; GGN, ground-glass nodule.

\section{Discussion}

We established a reliable procedure for confirming during surgery whether or not the resection line had been appropriate without cutting the resected specimen, even for impalpable tumors. We must occasionally make multiple sections from resected specimens if the tumor is impalpable, which compromises the subsequent pathological examination of the invasiveness of primary lung adenocarcinoma as well as the accurate measurement of the surgical margin. In our current initial clinical experience, the presence of an intrapulmonary small lesion (consolidation diameter $\leq 1 \mathrm{~cm}$ ) within the resected specimen was able to be confirmed in all patients, including two with lesions representing purely groundglass attenuation (Case 1 and 2). Furthermore, the surgical margin length was able to be accurately measured. The 

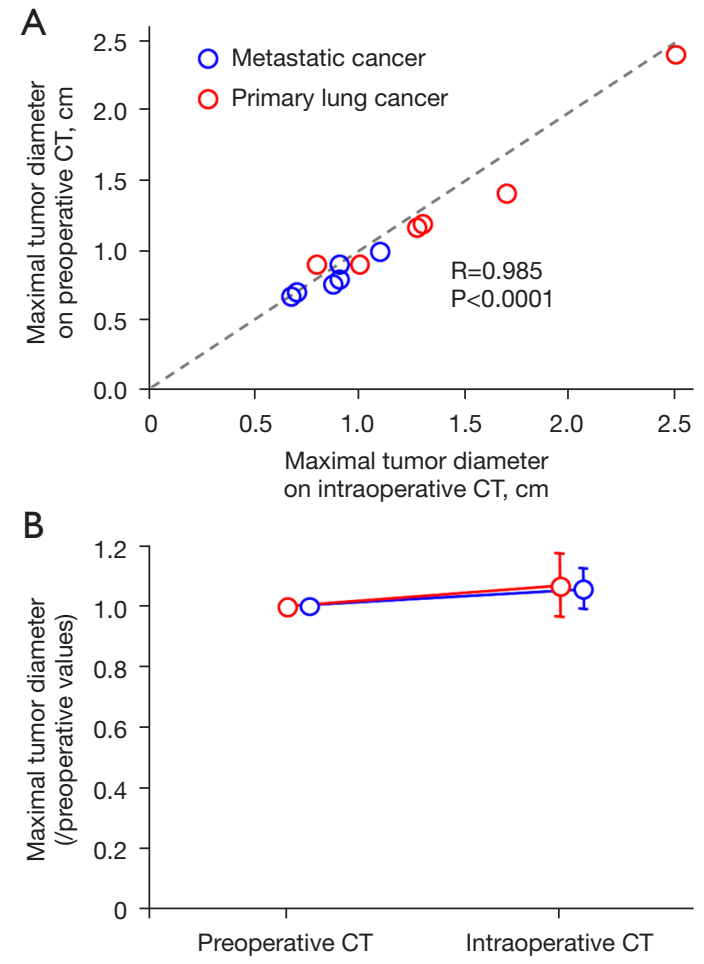

Figure 4 The correlation between the maximal tumor diameter on preoperative computed tomography $(\mathrm{CT})$ and on intraoperative CT in patients with primary lung cancer (red circle) or metastatic lung cancer (blue circle). The values correlated closely in both patients with primary lung cancer and metastatic lung cancer according to a correlation analysis (A). According to a paired t-test, the maximal tumor diameter on preoperative CT was not significantly different from that on intraoperative CT in both patients with primary lung cancer and metastatic lung cancer (B). The dotted line represents $y=x$.

further accumulation of data concerning the surgical margin as assessed via intraoperative CT, together with information on the prognostic outcomes, will provide reliable evidence regarding the necessary surgical margin for small lung cancer, which still remains controversial.

Pulmonary sublobar resection has become increasingly frequently employed in the field of thoracic surgery due to the increasing incidence of small lung adenocarcinoma possessing ground-glass attenuation. Pulmonary sublobar resection can be performed as radical surgery in some patients with predominant ground-glass lesions with a consolidation diameter of less than a certain value ( 5 to $10 \mathrm{~mm}$ ) $(8-10)$. However, even in cases eventually diagnosed as noninvasive adenocarcinoma, local recurrence can occur in
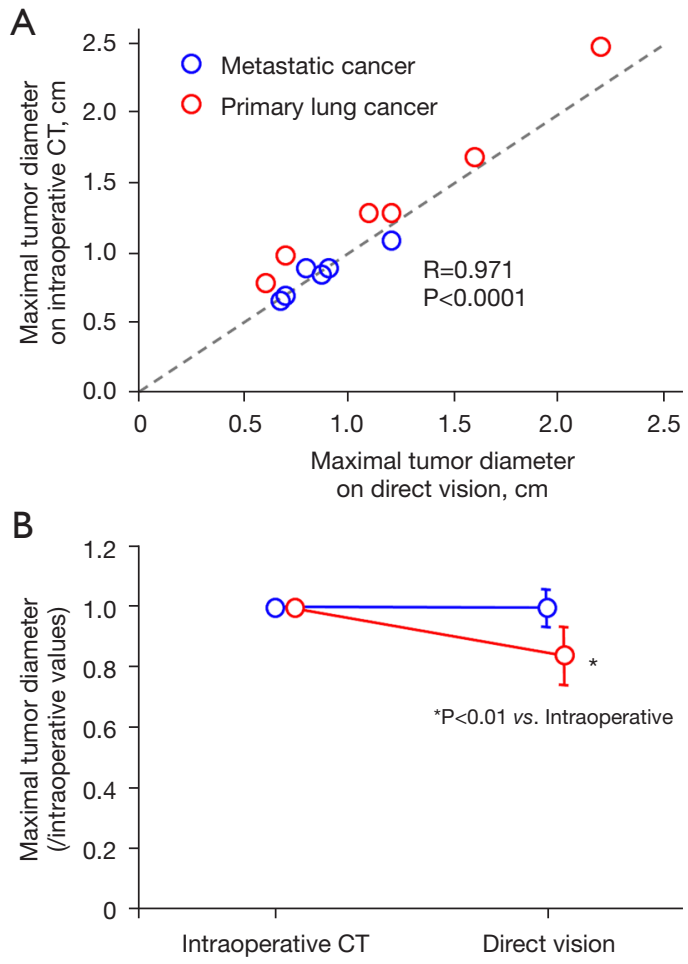

Figure 5 The correlation between the maximal tumor diameter on intraoperative computed tomography (CT) and on direct vision of the resected specimen in patients with primary lung cancer (red circle) or metastatic lung cancer (blue circle). Although the values correlated closely in overall patients according to a correlation analysis (A), the maximal tumor diameter on direct vision was significantly lower than that on intraoperative CT in patients with lung cancer (red circle) (B). The dotted line represents $\mathrm{y}=\mathrm{x}$.

patients without a sufficient surgical margin: Yoshida et al. reported three $(12.5 \%)$ patients with possible delayed cutend recurrence among 24 patients who underwent wedge resection for non-invasive adenocarcinoma (11). Sublobar resection can be also performed in patients with a poor pulmonary function even if the patients have invasive lesions. However, sublobar resection is known to increase the risk of local recurrence in patients with consolidation-type lung cancer compared with lobectomy, even if the lesion appears to have been resected with a sufficient margin (12).

As described above, we routinely use rapid intraoperative irrigation cytology as a substitute for surgical stump cytology. Because positive cytology result revealed to increase local recurrence $(4,13)$, we believe that 
A

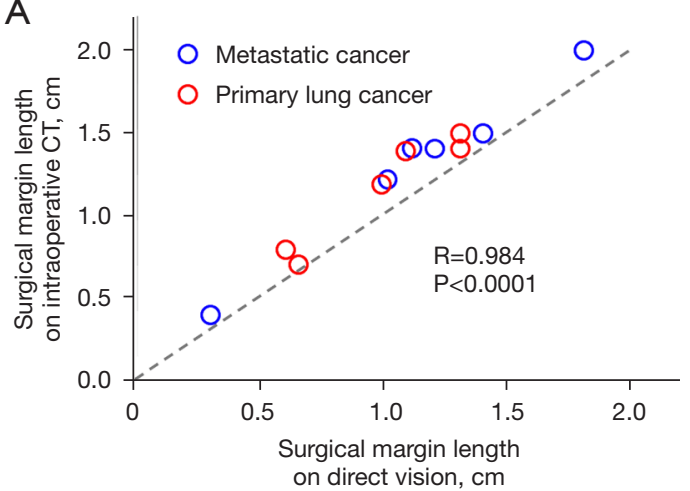

B

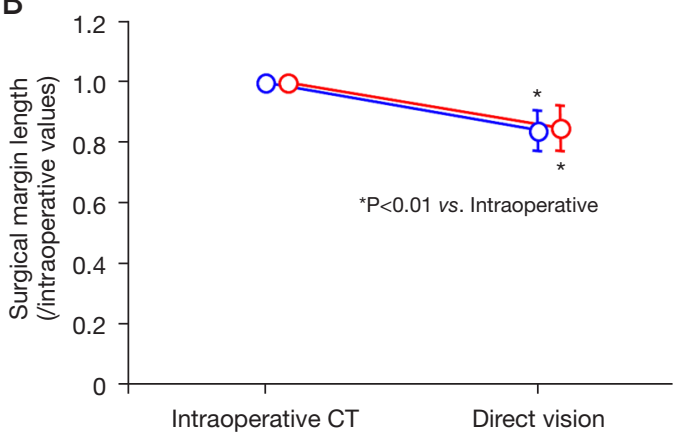

Figure 6 The correlation between the surgical margin on intraoperative computed tomography (CT) and on direct vision of the resected specimen in patients with primary lung cancer (red circle) or metastatic lung cancer (blue circle). Although the values correlated closely in overall patients according to a correlation analysis (A), the surgical margin on direct vision was significantly shorter than that on intraoperative CT in both patients with primary lung cancer (red circle) and those with metastatic lung cancer (blue circle) (B). The dotted line represents $y=x$.

intraoperative stump cytology may facilitate accurate intraoperative decision-making for additional surgical procedures (e.g., additional deep wedge resection or lobectomy).

One of the most important benefits of the present method is that we can measure the distance between the intrapulmonary lesions and any staple line (Figure 2) on multiplanar reconstruction images. This means that we can create multiple section images of the resected specimens without cutting the specimen itself, which can aid in determining how to make additional cut lines during performing additional resection for insufficient surgical margin.

According to the current quantitative assessment of the maximal tumor diameter, which was measured preoperatively, intraoperatively, and postoperatively, the diameters of the metastatic lesions, represented as purely solid nodules, were similar among the three groups. In contrast, the tumor apparently shrank in cases of primary lung cancer, represented as a ground-glass nodule, after formalin fixation $(84.0 \% \pm 9.6 \%$ of intraoperative values, $\mathrm{n}=6$ ). Likewise, the surgical margin apparently shrank among overall patients $(84.6 \% \pm 6.8 \%$ of intraoperative values, $n=12$ ). Thus, the pathologically determined surgical margin should be distinguished from the intraoperatively measured one.

Troschel et al. previously evaluated the resected specimens with micro-CT after pulmonary wedge resection for lung nodules (14). They correlated the micro-CT findings with pathological findings. Unfortunately, the ground glass lesions were not apparently depicted on microCT, which compromised accurate measurement of surgical margin length, because they did not inflate the resected specimens with air. We believe that micro-CT cannot be done, in practice, during surgery because scanning with micro-CT may took considerably longer time, as compared with conventional helical CT. Thus, our method is useful in intraoperative immediate and accurate evaluation of surgical margin, particularly in tumors with ground glass component.

There are some possible limitations in this study. First, small sample size could limit identification of technical pitfalls of the current procedure. Second, we did not evaluate long-term postoperative outcome regarding local recurrence, which may validate the length of surgical margin in our patients. However, rapid intraoperative irrigation cytology, as a substitute for surgical stump cytology, was negative in all 12 patients. Nonetheless, large scale study with long-term postoperative follow-up will clarify these issues.

\section{Conclusions}

Given the above findings, we believe that intraoperative CT is reliable for diagnosing the presence of an intrapulmonary tumor within the resected specimen as well as for measuring the minimal surgical margin necessary, particularly in patients undergoing sublobar resection for impalpable or ground-glass nodules.

\section{Acknowledgments}

The authors wish to acknowledge Tomohiro Kamikawa (RT) 
for his help in interpreting the significance of the imaging study. We also thank Dr. Michiyo Higashi for macroscopic and pathological assessment of the tumors.

Funding: None.

\section{Footnote}

Reporting Checklist: The authors have completed the MDAR checklist. Available at https://dx.doi.org/10.21037/ qims-21-562

Conflicts of Interest: All authors have completed the ICMJE uniform disclosure form (available at https://dx.doi. org/10.21037/qims-21-562). The authors have no conflicts of interest to declare.

Ethical Statement: The authors are accountable for all aspects of the work in ensuring that questions related to the accuracy or integrity of any part of the work are appropriately investigated and resolved. The study was conducted in accordance with the Declaration of Helsinki (as revised in 2013). This study was approved by the institutional review board of Kagoshima University Graduate School of Medical and Dental Sciences (Approval No. 210025), and individual consent for this retrospective analysis was waived.

Open Access Statement: This is an Open Access article distributed in accordance with the Creative Commons Attribution-NonCommercial-NoDerivs 4.0 International License (CC BY-NC-ND 4.0), which permits the noncommercial replication and distribution of the article with the strict proviso that no changes or edits are made and the original work is properly cited (including links to both the formal publication through the relevant DOI and the license). See: https://creativecommons.org/licenses/by-nc-nd/4.0/.

\section{References}

1. Sato M, Kuwata T, Yamanashi K, Kitamura A, Misawa K, Imashimizu K, Kobayashi M, Ikeda M, Koike T, Kosaka S, Fukai R, Sekine Y, Isowa N, Hirayama S, Sakai H, Watanabe F, Nagayama K, Aoyama A, Date H, Nakajima J. Safety and reproducibility of virtual-assisted lung mapping: a multicentre study in Japan. Eur J Cardiothorac Surg 2017;51:861-8.

2. Dendo S, Kanazawa S, Ando A, Hyodo T, Kouno Y, Yasui K, Mimura H, Akaki S, Kuroda M, Shimizu N, Hiraki Y.
Preoperative localization of small pulmo- nary lesions with a short hook wire and suture system: experience with 168 procedures. Radiology 2002;225:511-8.

3. Sawabata N, Ohta M, Matsumura A, Nakagawa K, Hirano H, Maeda H, Matsuda H; Thoracic Surgery Study Group of Osaka University. Optimal distance of malignant negative margin in excision of nonsmall cell lung cancer: a multicenter prospective study. Ann Thorac Surg 2004;77:415-20.

4. Sawabata N, Matsumura A, Ohota M, Maeda H, Hirano H, Nakagawa K, Matsuda H; Thoracic Surgery Study Group of Osaka University. Cytologically malignant margins of wedge resected stage I non-small cell lung cancer. Ann Thorac Surg 2002;74:1953-7.

5. Mohiuddin K, Haneuse S, Sofer T, Gill R, Jaklitsch MT, Colson YL, Wee J, Bueno R, Mentzer SJ, Sugarbaker DJ, Swanson SJ. Relationship between margin distance and local recurrence among patients undergoing wedge resection for small $(\leq 2 \mathrm{~cm})$ non-small cell lung cancer. J Thorac Cardiovasc Surg 2014;147:1169-75; discussion 1175-7.

6. Masuda D, Nishida T, Akiduki K, Tei Y, Fuji Y, Iwasaki M. Percutaneous computed tomography-guided lung needle marking without thoracocentesis for small-sized peripheral lung leasions. The Journal of the Japanese Association for Chest Surgery 2001:25;604-10.

7. Miyoshi T, Yoshida J, Aokage K, Tane K, Ishii G, Tsuboi $M$. Stapling cartridge lavage cytology in limited resection for pulmonary malignant tumors: assessment of cytological status of the surgical margin. Heliyon 2019;5:e01240.

8. Suzuki K, Koike T, Asakawa T, Kusumoto M, Asamura H, Nagai K, Tada H, Mitsudomi T, Tsuboi M, Shibata T, Fukuda H, Kato H; Japan Lung Cancer Surgical Study Group (JCOG LCSSG). A prospective radiological study of thin-section computed tomography to predict pathological noninvasiveness in peripheral clinical IA lung cancer (Japan Clinical Oncology Group 0201). J Thorac Oncol 2011;6:751-6.

9. Yamato $Y$, Tsuchida M, Watanabe T, Aoki T, Koizumi N, Umezu H, Hayashi J. Early results of a prospective study of limited resection for bronchioloalveolar adenocarcinoma of the lung. Ann Thorac Surg 2001;71:971-4.

10. Yoshida J, Nagai K, Yokose T, Nishimura M, Kakinuma R, Ohmatsu H, Nishiwaki Y. Limited resection trial for pulmonary ground-glass opacity nodules: fifty-case experience. J Thorac Cardiovasc Surg 2005;129:991-6.

11. Yoshida J, Ishii G, Yokose T, Aokage K, Hishida T, Nishimura M, Onuki T, Noguchi M, Nagai K. Possible 
delayed cut-end recurrence after limited resection for ground-glass opacity adenocarcinoma, intraoperatively diagnosed as Noguchi type B, in three patients. J Thorac Oncol 2010;5:546-50.

12. Ginsberg RJ, Rubinstein LV. Randomized trial of lobectomy versus limited resection for T1 N0 non-small cell lung cancer. Lung Cancer Study Group. Ann Thorac Surg 1995;60:615-22; discussion 622-3.

13. Sawabata N, Maeda H, Matsumura A, Ohta M, Okumura M; Thoracic Surgery Study Group of Osaka University. Clinical implications of the margin cytology findings

Cite this article as: Kamimura G, Ueda K, Suzuki S, Maeda K, Hakamada H, Sato M. Intraoperative computed tomography of a resected lung inflated with air to verify safety surgical margin. Quant Imaging Med Surg 2022;12(2):12811289. doi: 10.21037/qims-21-562 and margin/tumor size ratio in patients who underwent pulmonary excision for peripheral non-small cell lung cancer. Surg Today 2012;42:238-44.

14. Troschel FM, Gottumukkala RV, DiCorpo D, Mario J, Ott HC, Wright CD, Muniappan A, Lanuti M, Yang K, Shepard JO, Nardi V, Michaelson JS, Hariri LP, Fintelmann FJ. Feasibility of Perioperative MicroComputed Tomography of Human Lung Cancer Specimens: A Pilot Study. Arch Pathol Lab Med 2019;143:319-25. 